\title{
Médiévales
}

Langues, Textes, Histoire

48 | printemps 2005

Princes et princesses à la fin du Moyen Âge

\section{Alain Boureau, Satan hérétique. Naissance de la démonologie dans l'Occident médiéval (1280-1330)}

Paris, Odile Jacob, 2004, 318 p.

\section{Martine Ostorero}

\section{(2) OpenEdition}

\section{Journals}

Édition électronique

URL : https://journals.openedition.org/medievales/1087

DOI : 10.4000/medievales. 1087

ISSN : 1777-5892

\section{Éditeur}

Presses universitaires de Vincennes

Édition imprimée

Date de publication : 1 juin 2005

Pagination : 165-168

ISBN : 2-84292-169-0

ISSN : 0751-2708

\section{Référence électronique}

Martine Ostorero, «Alain Boureau, Satan hérétique. Naissance de la démonologie dans l'Occident médiéval (1280-1330)», Médiévales [En ligne], 48 I printemps 2005, mis en ligne le 02 décembre 2005, consulté le 23 avril 2022. URL : http://journals.openedition.org/medievales/1087 ; DOI : https:// doi.org/10.4000/medievales. 1087

Ce document a été généré automatiquement le 23 avril 2022

Tous droits réservés 


\section{Alain Boureau, Satan hérétique. Naissance de la démonologie dans l'Occident médiéval (1280-1330)}

Paris, Odile Jacob, 2004, 318 p.

\section{Martine Ostorero}

1 L'un des buts du nouvel ouvrage d'Alain Boureau est de montrer comment la chasse aux sorcières qui émerge dès 1430 est en continuité avec la démonologie scolastique développée un siècle plus tôt, entre 1280 et 1330. La rationalité scolastique, par son travail constant d'interrogation et d'exploration intellectuelle, a ouvert de dangereux champs de réflexion, en redéfinissant les rapports entre l'homme et les démons : elle a en partie rendu possible l'émergence du sabbat. L'hypothèse de l'auteur est que «le nouveau souci des démons serait né de la conjonction, de l'actualisation et de l'interaction de deux thèmes anciens, celui du pacte conclu avec le diable et celui de la possession» (p.13). Il examine dans son ouvrage ces deux pistes principales, qui ouvrent à leur tour sur une multitude de problématiques, dont certaines avaient déjà été proposées dans Médiévales au printemps 2003, ou dans d'autres articles.

2 C'est au pape Jean XXII (1316-1334) que l'on doit l'élaboration des outils intellectuels utilisés plus tard pour la chasse aux sorcières; il pose les bases d'une nouvelle perception de la magie et des savoirs qui ont pu lui être associés, comme l'astrologie ou l'alchimie. La bulle Super illius specula (1326 ou 1327) énonce pour la première fois que certaines pratiques magiques (fabrication d'images, d'anneaux ou de miroirs) dérivent directement de l'invocation des démons et que, de fait, les personnes qui se livrent à de tels actes encourent les peines réservées aux hérétiques. La magie, mais aussi indirectement les sortilèges, sont dès lors considérés comme des hérésies, et ceux qui sont soupçonnés de s'y livrer peuvent tomber dans le ressort de l'inquisition. Les rapports avec les démons entrent dans le champ des faits réels et ne sont plus des illusions, tels les rêves de vol nocturne du vieux canon Episcopi. La définition de Jean XXII du fait hérétique (factum hereticale) est capitale, dans la mesure où elle implique que l'hérésie n'est plus seulement une affaire d'opinion ou de croyance erronée : elle se 
manifeste par des actions - elle fait quelque chose. C'est bien sur ce terreau que se développe le sabbat des sorcières dans les années 1430 : pensons aux efforts continus des inquisiteurs et des démonologues pour chercher à tout prix - et en vain - des preuves matérielles de la réalité des rencontres sabbatiques.

3 La bulle Super illius specula est le résultat des commissions d'enquêtes judiciaires et doctrinales mandatées par Jean XXII en 1320 pour examiner la qualification comme hérésie de l'invocation des démons par les magiciens. Alain Boureau vient d'ailleurs de publier l'intégralité du dossier sous le titre Le Pape et les sorciers. Une consultation de Jean XXII sur la magie en 1320 (manuscrit B.A.V Borghese 348), Rome, École française de Rome, 2004. L'originalité de l'un des experts, Enrico del Carretto, est de démontrer la réalité du maléfice opéré par l'image, qui rend celui-ci efficace; le diable est présent dans l'image comme le signifié dans le signe. Le modèle de l'eucharistie permet à l'auteur de définir un sacrement satanique, basé sur la notion de pacte qui fonde la relation entre l'homme et le démon. Or, cette conception contractuelle des sacrements se développe dans la théologie scolastique dès 1240 avec la théorie de la causalité sacramentelle.

4 Alain Boureau propose dans un chapitre suivant un réexamen de la notion de pacte avec le diable, central dans la démonologie et la chasses aux sorcières. Il distingue un pacte faible, qui peut être renégociable ou faire l'objet d'un désaveu ou d'une restauration, d'un pacte fort, qui engage complètement le sujet et comporte une dimension sacramentelle et surnaturelle, mettant en cause l'ordre divin. L'histoire bien connue du pacte de Théophile, souvent évoquée comme source du pacte des sorciers, relève essentiellement du premier type et ne rend pas compte de la pertinence contextuelle du pacte. En faisant converger des éléments de culture marchande (convention d'échanges), des techniques juridiques (la notion de contrat et de personne), et une conception fonctionnelle et immanente du pouvoir politique (sorte de contrat social, de type vicarial ou conditionnel), l'auteur montre comment est perçue l'efficacité réelle du pacte conclu entre les hommes au XIII ${ }^{\mathrm{e}}$ siècle.

Un chapitre central présente ensuite les débuts de la démonologie scolastique. Le De malo de Thomas d'Aquin, rédigé à la fin de sa vie, forme un corps de doctrine ample et original, qui a suscité de nombreuses attaques, notamment de la part des milieux franciscains (Guillaume de la Mare, Pierre de Falco et Pierre de Jean Olivi). Les oppositions spirituelles et spéculatives s'articulent autour de la question de la nature et de la corporéité des démons, ainsi que des notions de libre-arbitre et de volonté au moment de leur chute. Si Thomas n'accorde qu'une faible capacité et vivacité aux démons, ceux-ci seront "libérés» par les franciscains, rencontrant leurs préoccupations eschatologiques. $\mathrm{Au} \mathrm{XV}^{\mathrm{e}}$ siècle, les démonologues de la chasse aux sorcières, de Nicolas Jacquier à Henri Institoris, n'oublieront pas cet héritage scolastique et intègreront le sabbat des sorcières dans ces cadres doctrinaux.

6 Autre piste pour repérer le tournant démonologique des années 1280-1330, les procès de canonisation. Après examen de cinq cas majeurs (Thomas de Cantiloupe, Louis d'Anjou, Nicolas de Tolentino, Pierre de Morrone et Chiara de Montefalco), l'auteur observe deux tendances : la première est à l'effacement des possédés au profit des fous, en raison d'un filtrage opéré par la Curie qui tend à éviter la qualification de démoniaque, au moment où se médicalise la folie. Mais les cas de possession ne disparaissent pas pour autant. Ils prennent des accents particuliers, comme en témoigne l'enquête sur Nicolas de Tolentino (1245-1305) : les possédées du couvent de Santa Lucia invoquent et appellent à l'aide le démon Bélial, comme dans un pacte 
conclu avec lui ; actives et non plus passives, elles se livrent à des gestes démoniaques qui sont l'inverse des actes chrétiens; enfin, ces possédées voient des démonsrevenants, qui sont des personnes jadis brûlées pour leurs méfaits, des hérétiques ou des morts sans confession. S'observent ainsi la présence renouvelée des démons dans les cœurs et les corps des hommes, et de nouvelles formes de possession qui associent Satan aux revenants, au moment même où Jean XXII redéfinit les rapports entre la magie, l'hérésie et la démonologie.

7 Autre figure de l'aliénation, vite démonisée, celle du somnambule, examinée à l'avantdernier chapitre : cette figure permet l'exploration des failles ouvertes dans l'édifice de la personnalité humaine, en montrant sa fragilité et sa capacité de dédoublement. En 1312, dans le canon Si furiosus, Clément $\mathrm{V}$ dégage le somnambule de toute responsabilité pénale, au même titre que l'enfant ou le fou; le sommeil est une sorte d'infirmité qui jette l'homme hors de son esprit, le plaçant dans un état de pure nature, disponible alors à une possession externe. Les discussions scolastiques autour des rapports entre l'âme et le corps - la théorie de l'âme comme forme substantielle du corps dans le thomisme, s'opposant à celle de la pluralité des formes substantielles chez les néoaugustiniens - contribuent à l'élaboration d'une nouvelle psychologie dans laquelle est redéfinie l'autonomie du sujet et l'unité de la personne humaine. Est admise la possibilité d'une personnalité double ou multiple, qui fait cohabiter dans le même corps l'âme de l'individu et un hôte divin ou satanique. Ainsi, «l'anthropologie scolastique, en explorant les limites de l'action et de la conscience, avait décrit les zones de vide ou de fragilité de la personnalité humaine» (p. 227). Une fragilité que les démons vont exploiter à l'époque des sorcières.

Dès la seconde moitié du XIII ${ }^{\mathrm{e}}$ siècle, à l'ère du soupçon suivant la formule d'André Vauchez, il devient de plus en plus difficile de distinguer les illusions démoniaques des visions inspirées par Dieu. À la place de l'antique don de discernement des esprits, les clercs sont contraints de développer des techniques fondées sur l'observation méticuleuse des phénomènes de transes, d'ascèses ou de convulsions. L'essor de la mystique, avec le thème du ravissement divin, présente le miroir de la possession diabolique, en raison de la similitude des modes d'action des spiritus, esprits divins ou démoniaques. Dans un dernier chapitre, deux formes de possession divine sont présentées, que l'auteur désigne sous les termes d'incorporation et d'inhabitation: le cas de Chiara de Montefalco, qui reproduit en elle le divin en formant en son cœur l'imitation matérielle et parfaite des instruments de la Passion; celui d'Angèle de Foligno, qui fait parler en elle et avec elle le Christ et le Saint-Esprit. Des cas qui mettent en lumière dans l'anthropologie de la personne la faculté d'imagination et sa capacité d'action sur le corps, un thème que l'on retrouve aussi au cœur des discussions démonologiques $\mathrm{du} \mathrm{Xv}^{\mathrm{e}}$ siècle, au moment du plein développement du sabbat des sorcières.

9 Au terme de l'exploration de ces différentes pistes, l'auteur constate que vers 1320 les démons sont dotés de nouveaux modes d'actions et de relations avec les hommes - tels le pacte et le sacrement satanique -, et qu'ils sont capables de détourner n'importe quelle personne, désormais fragilisée et perméable aux influences surnaturelles, dans un contexte où de fortes tendances eschatologiques désignaient les signes annonciateurs de la libération des démons. Un siècle avant l'essor de la chasse aux sorcières, ce peuple des démons semble déjà prêt à fondre sur les humains. 
10 Si la doctrine contre les magiciens et les sorciers semble être aboutie vers 1330, pourquoi n'est-ce qu'un siècle plus tard que l'on rencontre les premières sectes de sorciers adorateurs du diable, infanticides et cannibales? Pour Alain Boureau, ce «retard à l'allumage » serait dû à la réticence de la papauté à déléguer son pouvoir d'enquête à l'inquisition. C'est «l'abandon forcé de l'absolutisme pontifical, après les conciles de Constance et de Bâle, qui ouvrit les premières campagnes judiciaires contre les sorciers et les adorateurs de démons » (p. 59).

11 On le constate, l'ouvrage d'Alain Boureau n'est pas à proprement parler une « histoire de la démonologie», comme l'indique le sous-titre de couverture, mais une vaste enquête au cours de laquelle l'auteur traque le démon à travers différents discours (débats scolastiques, expertises théologiques ou procès de canonisation) et différents champs (hérésie, magie, mystique, somnambulisme et possession), ouvrant sur des domaines inhabituels dans cette thématique, tels la théologie sacramentelle, la théorie des contrats et l'anthropologie de la personne humaine. Ces pistes foisonnantes convergent à un moment particulier, les années 1280-1330, soit celles des pontificats de Clément $\mathrm{V}$ et de Jean XXII, que l'auteur considère à juste titre comme un tournant démonologique.

Il reste peut-être encore quelques trous dans cette histoire de la démonologie, qui pourraient expliquer ce retard d'un siècle à l'allumage des bûchers : comment s'est constituée l'image d'un diable à la forte corporéité, avec lequel le sorcier est en relation directe au sabbat (dialogues et coïts démoniaques sont des preuves de sa réalité physique dans le monde d'ici-bas)? Comment est-on passé du démon familier qui répond aux invocations des magiciens, tel celui de Boniface VIII, à un diable qui trône au sabbat tel un seigneur et un autre dieu, qui juge et domine ses sujets soumis à une obéissance absolue et unilatérale (l'apostasie exclut définitivement toute relation avec le divin)? Le sabbat des sorcières dessine une conception du diable qui dépasse celle élaborée par les scolastiques, et qui est le fruit de nouveaux développements survenus dans la démonologie et l'hérésie dans la seconde moitié du XIve siècle.

13 Enfin, l'auteur exclut de cette histoire menant à la chasse aux sorcières la notion de secte, qui paraît pourtant fondamentale. Sans l'expliquer, il semble même éviter volontairement le terme, au profit de la formule de "réseau hérétiques et jurés de complices de Satan » (p. 263). Or, la notion de secte est bien ancrée dans la tradition de l'hérésie; elle souligne l'aspect collectif des pratiques et des croyances des hérétiques et va être progressivement diabolisée au cours du XIV siècle. Elle prend un accent nouveau dans une bulle promulguée par Alexandre $V$ en 1409 qui dénonce les "nouvelles sectes", assorties de "rites prohibés ", inventés par des juifs et chrétiens, sorciers, devins ou invocateurs de démon. $A u \mathrm{XV}^{\mathrm{e}}$ siècle, la secte ne réunit plus uniquement des hérétiques facilement reconnaissables, mais elle peut agréger tout le monde sous la tutelle du diable. Différents types de sectes, qu'elles soient réelles comme les cathares, les vaudois ou les hussites ou plus imaginaires, telles les Lucifériens, les adeptes du Libre Esprit et enfin les sorciers, ont aussi contribué à l'élaboration du sabbat des sorcières. Les sectes hérétiques pourraient être le chaînon manquant entre la démonologogie de Jean XXII et le sabbat du XV siècle.

14 L'immense mérite de l'ouvrage d'Alain Boureau est de donner accès à un champ parfois délaissé par les historiens intéressés par la sorcellerie. Ces derniers se sont montrés davantage préoccupés par la dimension judiciaire des chasses aux sorcières (les questions de procédure, la redoutable machine inquisitoriale, l'aveu et la torture), par 
leur insertion dans un contexte politique et religieux et leur instrumentalisation par les instances dirigeantes, ou encore par les liens entre culture populaire et savante, tels qu'ils sont perceptibles dans les aveux des accusés, et ils ont en effet peu pris en compte les élaborations spéculatives des scolastiques en matière de démonologie. Désormais, ce domaine, cher à l'auteur, va pouvoir trouver sa place dans la réflexion sur le sabbat des sorcières, lui offrant des pistes de recherches très stimulantes. 